\title{
High Affinity Mannotetraose as an Alternative to Dextran in ConA Based Fluorescent Affinity Glucose Assay Due to Improved FRET Efficiency
}

\author{
Andrea K. Locke ${ }^{\star}$, Brian M. Cummins ${ }^{\ddagger}$, and Gerard L. Coté \\ Department of Biomedical Engineering, Texas A\&M University, College Station, Texas 77843, \\ United States \\ ¥Department of Biomedical Engineering, University of North Carolina at Chapel Hill and North \\ Carolina State University, Raleigh, North Carolina 27695, United States
}

\section{Abstract}

Diabetes mellitus affects millions of people worldwide and requires that individuals tightly selfregulate their blood glucose levels to minimize the associated secondary complications. Continuous monitoring devices potentially offer patients a long-term means to tightly monitor their glucose levels. In recent years, fluorescent affinity sensors based on lectins (e.g., Concanavalin A (ConA)) have been implemented in such devices. Traditionally, these sensors pair the lectin with a multivalent ligand, like dextran, in order to develop a competitive binding assay that changes its fluorescent properties in response to the surrounding glucose concentrations. This work introduces a new type of fluorescent ligand for FRET-based assays in an attempt to improve the sensitivity of such assays. This ligand is rationally designed to present a core trimannose structure and a donor fluorophore in close proximity to one another. This design decreases the distance between the FRET donor and the FRET acceptors on ConA to maximize the FRET efficiency upon binding of the ligand to ConA. This work specifically compares the FRET efficiency and sensitivity of this new competing ligand with a traditional dextran ligand, showing that the new ligand has improved characteristics. This work also tested the long-term thermal stability of the assay based on this new competing ligand and displayed a MARD of less than $10 \%$ across the physiological range of glucose after 30 days incubation at $37^{\circ} \mathrm{C}$. Ultimately, this new type of fluorescent ligand has the potential to significantly improve the accuracy of continuous glucose monitoring devices based on the competitive binding sensing approach.

\section{Graphical abstract}

\footnotetext{
*Corresponding Author: andrealocke88@ tamu.edu. Supporting Information The Supporting Information is available free of charge on the ACS Publications website at DOI: 10.1021/acssensors.5b00304. Author Contributions The manuscript was written through contributions of all authors.

Notes

The authors declare no competing financial interest.
} 


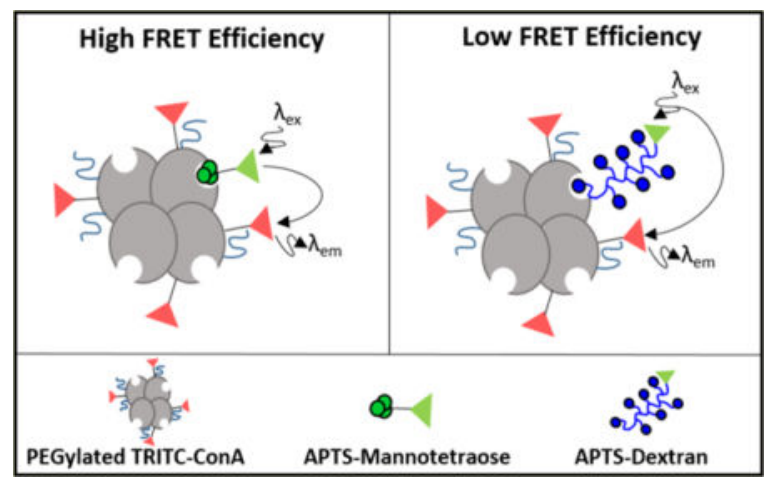

\section{Keywords}

glucose sensing; competing ligand; mannotetraose; dextran; continuous glucose sensing; competitive binding assays

Diabetes is a disease that disrupts the body's normal regulation of blood glucose levels, and proper self-management is required to reduce the risks of secondary complications (i.e., gangrene, heart disease, renal failure, etc.). For people with Type-1 Diabetes, this management consists of measuring one's blood glucose and delivering appropriate levels of insulin to return to normal levels. Continuous glucose monitoring (CGM) has been shown to decrease secondary complications, and improved CGM devices have the potential to alleviate the demand on the user even further (e.g., artificial pancreas). ${ }^{1-4}$

The use of competitive binding assays for glucose sensing was introduced in the 1980s by Meadows and Schultz, and Schultz later demonstrated its use as an implantable affinitybased glucose sensor. ${ }^{5,6}$ There are now several companies developing CGM devices based on this technology to achieve improved sensitivity, accuracy, and working lifetime. ${ }^{7}{ }^{9}$ Also, more recently research conducted by Ballerstadt et al. led to the development of a fiber coupled CGM device, currently under license with BioTex Inc. as a second-generation Fluorescence Affinity Sensor (FAS). In vivo human studies recently conducted on this device showed a mean absolute relative error of $13 \%$ over a period of $4 \mathrm{~h} .{ }^{10}$ In addition, Muller et al. in 2013 introduced another fiber coupled CGM device called FiberSense. This device showed improved MARD percentage over a longer period of time (14 days). ${ }^{11}$ Both FiberSense and the second-generation FAS are transdermal CGM devices which have the possible disadvantage of irritation at the implant site due to continuous movement of the transdermal optical fiber. The PreciSense A/S device proposed by Nielsen et al. and recently acquired by Medtronic has the potential to overcome this disadvantage due to it being a fully implanted degradable microsensor. In a preclinical in vivo study, this microsensor displayed in a MARD of $11.4 \%$ over a period of $70 \mathrm{~h} .{ }^{12}$ All three devices can be categorized as fluorescent affinity sensors and are based on a competitive binding assay.

These fluorescent, competitive binding assays contain a glucose receptor paired with a glucose-like competing ligand. ${ }^{7,13,1}$ The components can be fluorescently labeled, and the binding equilibrium of this pair is designed to be dependent on the glucose concentration in the system. Changes in the fluorescence properties (e.g., anisotropy, lifetime, and intensity) 
at equilibrium can be translated into changes in glucose concentration. Herein, Förster Resonance Energy Transfer (FRET) has been identified as an ideal transduction mechanism to observe changes in the optical properties of the assay in response to concentration changes. ${ }^{15}$

In brief, FRET is a phenomenon where the energy of an excited fluorophore can nonradiatively transfer its energy to another fluorophore. With the properly chosen donor and acceptor fluorophores (overlapping emission/excitation spectra), the donor transfers its energy when the acceptor fluorophore is brought into close proximity (eq 1$)$. In this equation, $E$ is the FRET efficiency, and $R$ and $R_{0}$ are the distance between the two fluorophores and the Forster radius, respectively. ${ }^{16}$

$$
E=\frac{1}{1+\left(\frac{R}{R_{0}}\right)^{6}}
$$

Typically, FRET-based competitive binding assays employ lectins as the glucose receptor. Concanavalin A (ConA) and mannan binding lectin (MBL) have been used by BioTex and Medtronic, respectively. ${ }^{12,14}$ Concerns regarding the use of ConA for lectin-based glucose sensing have been addressed by Ballerstadt et al. where they concluded that the dosage of ConA proposed in the development of these sensors are much lower than the minimum dose required to observe any sign of cell toxicity. ${ }^{17}$ Furthermore, the group reported that the toxicity is lower than with glucose oxidase, which is currently being used in most CGM devices based on enzymatic glucose sensing. ${ }^{17}$

The competing ligand used in the competitive binding assay is chosen to have a higher affinity than glucose to achieve the desirable shift in equilibrium due to physiological glucose changes. This higher affinity has typically been achieved using a competing ligand that presents multiple glucose residues (e.g., dextran and glycosylated dendrimers). ${ }^{18,19}$ Furthermore, by labeling the competing ligand with a donor fluorophore and the receptor (e.g., ConA) with an acceptor fluorophore, the assay is designed to induce FRET when complexes are formed. The equilibrium of these complexes shifts accordingly to changes in glucose concentration, and this shift can be tracked by measuring the ratio of the peak fluorescence intensities of the two fluorophores.

We have recently shown that the long-term stability of the assay can be maintained by tethering ConA to PEG chains (i.e., PEGylating ConA) and introduced a new type of competing ligand that achieves the desirable affinity while preventing aggregation with free ConA in free solution. ${ }^{20,21}$ Also, ConA is known to bind to both glucose and mannose residues with different binding affinities. Therefore, the specificity of this modified ConA based assay was addressed in previous work, which showed the fluorescence anisotropy response of the assay in the presence of varying concentrations of D-glucose, D-galactose, and methyl- $\boldsymbol{a}$-D-mannopyranoside. ${ }^{22}$

Herein, we introduce a new type of fluorescently labeled competing ligand for use in FRETbased glucose sensing assays. This ligand is rationally designed to present a single binding 
epitope (core trimannose) in close proximity to the signaling fluorophore. Using traditional, multivalent ligands for comparison (two different sized dextrans), we show that this new type of ligand improves the FRET efficiency upon binding to acceptor-labeled lectin. We then display that this enabling advantage improves the sensitivity of the assay to changes in physiological glucose concentrations ( 0 to $400 \mathrm{mg} / \mathrm{dL}$ ) in comparison to the dextrans. Finally, we show that this new ConA-Mannotetraose based assay is thermally stable after incubation at body temperature $\left(37^{\circ} \mathrm{C}\right)$ for 30 days with a MARD of less than $10 \%$.

\section{EXPERIMENTAL SECTION}

\section{Materials}

Concanavalin A fluorescently labeled with tetramethylrhodamine (TRITC-ConA) lyophilized powder was purchased from Life Technologies (Grand Island, NY). Manganese (II) chloride $\left(\mathrm{MnCl}_{2}\right)$, trizma hydrochloride (Trizma-HCl), sodium bicarbonate, Dextran-2 $\mathrm{MDa}(\mathrm{Dex}-2 \mathrm{M})$, and methyl- $a$-D-mannopyranoside (MaM) were purchased from Sigma (St. Louis, MO), while Dextran-70 kDa (Dex-70k) was purchased from TCI America (Portland, Oregon). Dextrose (D-glucose) was purchased from Fisher Scientific (Hampton, New Hampshire). Methoxyl-poly(ethylene glycol)- $N$-hydroxylsuccinimide-succinimidyl carbonate (mPEG-NHS (SC), $5 \mathrm{kDa}$ ) was purchased from Nanocs (New York, NY). Calcium chloride dihydrate $\left(\mathrm{CaCl}_{2}\right)$ was obtained from J.T. Baker (Center Valley, PA), and sodium chloride $(\mathrm{NaCl})$ was from Mallinckrodt Chemical Inc. (St. Louis, MO). Aminopyrene trisulfonate mannotetraose (APTS-MT) was synthesized with Dr. Vigh at Texas A\&M University. The APTS labeling kit was purchased from Prozyme (Hayward, CA). The TRIS buffer ( $\mathrm{pH}$ 7.4, $10 \mathrm{mM}$ Trizma-HCl, $0.15 \mathrm{M} \mathrm{NaCl}, 1 \mathrm{mM} \mathrm{MnCl}$, and $1 \mathrm{mM} \mathrm{CaCl}_{2}$ ) and the sodium bicarbonate buffer $(\mathrm{pH} 8.5$ and $0.15 \mathrm{M} \mathrm{NaCl})$ were prepared with deionized water $(18 \mathrm{M} \Omega \mathrm{cm})$.

\section{Synthesis of Assay Components (i.e., APTS-MT, APTS-Dextran, and mPEG-TRITC-ConA)}

The synthesis of APTS-MT was performed using reductive amination and was previously discussed in detail. ${ }^{20}$ Briefly, $13.5 \mu \mathrm{L}$ of $1 \mathrm{M}$ APTS in acetic acid was added to $\sim 1 \mathrm{mg}$ of mannotetraose, allowing for the Schiff base intermediate to form between the reducing terminus of mannotetraose and APTS. To stabilize this into a covalent bond, $13.5 \mu \mathrm{L}$ of $1 \mathrm{M}$ sodium cyanoborohydride in tetrahydrofuran was added to the acetic acid mixture. This was well mixed and allowed to react at room temperature for $15 \mathrm{~h}$. The APTS-MT product was then purified via a HILIC column.

Two unique dextrans (MW: $70 \mathrm{kDa}$ and $2 \mathrm{MDa}$ ) were labeled with APTS using the same reductive amination approach. Dex-2 $\mathrm{M}$ allows for comparison with the proposed competing ligand due to similar binding affinities while Dex-70k is the most common competing ligand used in ConA based affinity assays. These fluorescent ligands (APTS-Dex-70k and APTSDex-2M) were synthesized using an APTS labeling kit from Prozyme. Briefly, stock solutions of $50 \mathrm{mg} / \mathrm{mL}$ Dex-70k and $100 \mathrm{mg} / \mathrm{mL}$ Dex-2 M were prepared in DI water. Next, 10 and $20 \mu \mathrm{L}$ of these solutions (Dex-70k and Dex-2M, respectively) were added to new centrifuge tubes. These solutions were centrifuged and then dried under a vacuum in a desiccator for $6 \mathrm{~h}$. Separately, the APTS reagent solution was then prepared by mixing the 
APTS solution (2.4 $\mu \mathrm{L})$, APTS catalyst ( $6 \mu \mathrm{L})$, and reductant solution $(2.4 \mu \mathrm{L})$ from the kit. Then, APTS reagent solution ( $4 \mu \mathrm{L}$ ) was added to each dried dextran samples and mixed well. These samples were sealed and placed in a water bath at $37^{\circ} \mathrm{C}$ for $2 \mathrm{~h}$. After removing the solutions from the water bath, DI water $(500 \mu \mathrm{L})$ was added to each solution. The samples were then purified via $10 \mathrm{kDa}$ centrifugal filters (Nanocep from Pall) before passing through a GE Healthcare illustra MicroSpinTM G-25 column at 3000 rpm for $2 \mathrm{~min}$. An absorbance scan, from 240 to $600 \mathrm{~nm}$, of the purified solution was taken via a Tecan microplate reader to determine whether the sugar was conjugated to the dye. The estimated number of dyes per dextran was calculated using the sulfuric acid based method designed by Albalasmeh et al. (Figure S-1.1 and Figure S-1.2). ${ }^{23}$

The synthesis of mPEG-TRITC-ConA has been previously described in detail. ${ }^{21}$ Briefly, 10 mg of TRITC-ConA was dissolved in $\sim 1 \mathrm{~mL}$ sodium bicarbonate buffer. To occupy ConA's binding site, $2 \mathrm{mg}$ of MaM was added to the protein solution. Next, $\sim 25 \mathrm{mg}$ of mPEG-NHS $(5 \mathrm{kDa}$ ) was added to the solution for a molar concentration ratio of 16:1 mPEG to ConA. The solution was then continuously mixed for $6 \mathrm{~h}$. Afterward, the solution was allowed to incubate for another $18 \mathrm{~h}$ at room temperature without mixing. Dialysis against TRIS buffer removed MaM and unbound mPEG-NHS. The final concentration of mPEG-TRITC-ConA was determined by taking an absorbance scan from 240 to $640 \mathrm{~nm}$ using UV-vis spectrometer.

\section{Binding Affinity Studies}

The binding affinity of the three different competing ligands to mPEG-TRITC-ConA was determined by tracking the fluorescence emission spectra during independent titration studies. Stock solutions of mPEG-TRITC-ConA $(15.4 \mu \mathrm{M})$ and APTS-MT $(10 \mu \mathrm{M})$ were first prepared separately in TRIS buffer. In a $1 \mathrm{~cm}$ path length cuvette $(\mathrm{C} 1), 1 \mathrm{~mL}$ of the assay ( $3 \mu \mathrm{M}$ mPEG-TRITC-ConA and $200 \mathrm{nM}$ APTS-MT) was prepared in TRIS buffer from the stock solutions. Fluorescence emission (475 to $650 \mathrm{~nm}$ ) was measured with an ISS PC1 spectrofluorometer $\left(\lambda_{\mathrm{ex}} 450 \mathrm{~nm}\right)$. Subsequent emission scans were performed with the APTS-MT at the same concentration and decreased concentrations of mPEG-TRITC-ConA. Briefly, this was done by removing $500 \mu \mathrm{L}$ of assay solution from $\mathrm{C} 1$ and adding $500 \mu \mathrm{L}$ of $200 \mathrm{nM}$ APTS-MT in TRIS buffer. This provided a new assay that comprised $200 \mathrm{nM}$ APTS-MT and $1.5 \mu \mathrm{M}$ mPEG-TRITC-ConA. The solution was mixed thoroughly and allowed to re-equilibrate before measuring the fluorescence spectra. This method was repeated to achieve final concentrations of mPEG-TRITC-ConA of $750 \mathrm{nM}, 375 \mathrm{nM}$, and $187.5 \mathrm{nM}$. Separate fluorescent scans of $1 \mathrm{~mL}$ APTS-MT $(200 \mathrm{nM})$ and $1 \mathrm{~mL} \mathrm{mPEG}-$ TRITC-ConA $(3 \mu \mathrm{M})$ in TRIS buffer were taken under the same excitation conditions as the controls.

After all spectra were obtained from a given titration study, the fluorescence intensity at the peak emission for the donor fluorophore (i.e., APTS @ $520 \mathrm{~nm}$ ) was plotted against mPEGTRITC-ConA concentration. Each curve was then normalized to 1 at ConA $=0 \mathrm{nM}$ and fitted with eq 2, giving the association constant to ConA $\left(K_{\mathrm{a}}=1 / K_{\mathrm{d}}\right)$ for the given competing ligand. This fit also generated $F_{\infty}$, which is related to the efficiency of FRET transfer when the given competing ligand is bound to ConA. 


$$
F_{[\mathrm{ConA}]}=\left(\frac{1-F_{\infty}}{1+\frac{[\mathrm{ConA}]}{K_{\mathrm{d}}}}+F_{\infty}\right)
$$

This method was repeated to determine the binding affinity of APTS-Dex-2 M (52 nM) to mPEG-TRITC-ConA (0 to $850 \mu \mathrm{M}$ ). An attempt was made to perform this for APTSDex-70k; however, the results were inconclusive. This is because its affinity to ConA is too low to see an appreciable change in the donor fluorescence at these ConA concentrations.

\section{Determining and Comparing the FRET Efficiencies of the Assays}

The approximate FRET efficiencies for the different competing ligands when bound to ConA were calculated using the parameter from the previous fit $\left(F_{\infty}\right)$. Here, the value associated with the fluorescence of APTS-MT in the absence of ConA (100\%) can be used as a control where there is no FRET transfer. The value associated with the fluorescence of APTS-MT in the presence of infinite ConA $\left(F_{\infty}\right)$ indicates the percentage of the original fluorescence from APTS-MT when all APTS-MT molecules in solution are bound to ConA. Therefore, using eq 3 the average FRET Efficiency $(E)$ when bound to ConA can be calculated for each competing ligand. The average distance $(r)$ between the APTS and the TRITC when the competing ligand is bound to ConA can be approximated by using these calculated efficiencies and the approximate Forster Radius $\left(R_{0}\right) .{ }^{16}$

$$
\begin{gathered}
E=1-F_{\infty} \\
r=R_{0}\left(\frac{1}{E}-1\right)^{1 / 6}
\end{gathered}
$$

\section{Glucose Responses of Assays}

The glucose responses of the different individual assays were measured by tracking the change in fluorescence intensity of donor and acceptor fluorophore. Using the binding affinities estimated above, the concentration of mPEG-TRITC-ConA was optimized using eq 5 to allow for at least $50 \%$ of the assay to be in its bound state prior to the introduction of glucose.

$$
\% B=\frac{[\mathrm{ConA}]}{K_{\mathrm{d}}+[\mathrm{ConA}]}
$$

A stock glucose concentration of $39500 \mathrm{mg} / \mathrm{dL}$ was prepared in TRIS buffer. In a cuvette, 1 $\mathrm{mL}$ of the assay solution (200 $\mathrm{nM}$ APTS-MT and $1 \mu \mathrm{M}$ mPEG-TRITC-ConA) was prepared in TRIS buffer and mixed well. After giving sufficient time for the assay to reach 
equilibrium, the fluorescence emission was scanned ( $\lambda_{\mathrm{ex}} 450 \mathrm{~nm}, \lambda_{\mathrm{ex}}: 475-675 \mathrm{~nm}$ ).

Aliquots of the stock glucose were added to the assay to vary the glucose concentration across physiological concentrations. After each addition, the solution was mixed well and given sufficient time to equilibrate prior to scanning the fluorescence emission in the same manner. Fluorescence scans were recorded for glucose concentrations that ranged from 0 $\mathrm{mg} / \mathrm{dL}$ to $400 \mathrm{mg} / \mathrm{dL}$. This was done in triplicate for the APTS-MT assay and repeated for assays based on the APTS-Dex-2 M ligand (52 nM APTS-Dex-2 M and $3 \mu \mathrm{M}$ mPEGTRITC-ConA) as well as assays based on the APTS-Dex-70k ligand (200 nM APTSDex-70k and $25 \mu \mathrm{M}$ mPEG-TRITC-ConA). Higher concentrations of mPEG-TRITC-ConA were required in the APTS-Dex-70k assay because the affinity is lower for that competing ligand to bind to the receptor. After the glucose-dependent fluorescence emission curves were obtained for each assay, the fluorescence intensity ratio $\left(F_{520} / F_{600}\right)$ was calculated and plotted against the associated glucose concentration. The sensitivity of each assay was then calculated and compared. The data was then fitted to a Boltzmann curve to determine how well each assay can predict glucose concentration.

\section{Glucose Response of 30 days at $37^{\circ} \mathrm{C}$}

The thermal stability of the APTS-MT based assay was evaluated by determining its ability to predict changes in glucose concentration after incubation at body temperature $\left(37^{\circ} \mathrm{C}\right)$ for 30 days. In a $50 \mathrm{~mL}$ centrifuge tube, a stock glucose concentration of $10000 \mathrm{mg} / \mathrm{dL}$ was prepared in TRIS buffer. In a cuvette, $1 \mathrm{~mL}$ stock assay solution (200 nM APTS-MT and 1 $\mu \mathrm{M}$ mPEG-TRITC-ConA) was prepared in TRIS buffer. The assay was placed in an incubator at $37^{\circ} \mathrm{C}$ for 30 days. A spectrophotometer was then used to measure the fluorescence emission of the sample $\left(\lambda_{\mathrm{ex}} 450 \mathrm{~nm}\right)$. Next, fluorescence emission scans were taken for varying glucose concentrations $(0 \mathrm{mg} / \mathrm{dL}$ to $350 \mathrm{mg} / \mathrm{dL})$ by adding small aliquots of the stock glucose solution to the cuvette. Each scan was taken after an incubation period of 2 min following the introduction of glucose.

After the FRET curves were obtained, the ratio of fluorescence intensity $\left(F_{520} / F_{600}\right)$ was plotted against increasing glucose concentration and fitted to a typical competitive binding curve in MatLab. ${ }^{24}$ This curve fit was then use to determine and plot the predicted versus actual glucose concentration.

\section{RESULTS}

\section{Binding Affinity of Different Competing Ligands}

The glucose response of APTS-MT based assay was compared to that of an assay comprising dextran molecule with similar binding affinity (i.e., Dex-2M), to evaluate whether the structure and size of the newly introduced monovalent ligand plays a role in its improved performance. First, to ensure that the binding affinities were still comparable even after PEGylation, the titration curves generated previously were used to determine the $K_{\mathrm{a}}$. The plot of the fluorescence intensity, at the donor peak, against increasing concentration of mPEG-TRITC-ConA was fitted with eq 2 and the binding affinity was determined to be 3.2 $\times 10^{6} \mathrm{M}^{-1}$ and $2.61 \times 10^{6} \mathrm{M}^{-1}$ for APTS-MT and APTS-Dex-2M, respectively. Benzeval reported a similar binding affinity of $\sim 1.42 \times 10^{6} \mathrm{M}^{-1}$ for Dex-2 $\mathrm{M}$ binding to ConA and our 
previous fluorescence anisotropy work reported an affinity of $\sim 5 \times 10^{6} \mathrm{M}^{-1}$ for PEGlyated ConA-APTS-MT assay which validates our findings. ${ }^{21,25}$

These $K_{\mathrm{a}}$ values are on the same order; therefore, we can assume that any difference in the fluorescence glucose response will be primarily due to the differences in the distance of the donor fluorophore (APTS) to the acceptor (TRITC) based on the structure and size of the competing ligands.

\section{FRET Efficiency of Mannotetraose versus Dextran Based Assay}

The eventual performance of the competitive binding assay is dependent on the efficiency of FRET $(E)$ when the donor-labeled competing ligand is complexed with the acceptor-labeled ConA. This efficiency was evaluated for each APTS-labeled competing ligand using the parameters from the titration binding study.

APTS-MT is expected to have better FRET efficiency over the dextran based assays due to its size and the location of its single fluorophore on the competing ligand. The MW of mannotetraose is $\sim 1 \mathrm{kDa}$ compared to dextran (70 kDa and $2 \mathrm{M} \mathrm{Da})$. This much smaller size should ideally allow for its fluorophore to be brought closer into proximity to the fluorophores on ConA when bound compared to dextran.

The titration plots in Figure 1 show a greater change in fluorescence intensity for the APTSMT based assay compared to the APTS-dex- $2 \mathrm{M}$ based assay, over a slightly similar concentration range. After normalizing the fluorescence intensity at $520 \mathrm{~nm}$ and fitting it to eq 2, APTS-MT had a much higher FRET efficiency of $~ 89 \%$ compared to that of APTS-

Dex-2 M whose efficiency was determined to be $\sim 25 \%$. Furthermore, when compared to the traditional Dex-70k, APTS-MT also displayed improved efficiency. Moreover, due to its lower affinity, APT-Dex-70k required a much higher concentration range of the fluorescently labeled receptor required to obtain a very small and inconsistent FRET change making it difficult to determine its exact FRET efficiency.

The average distance between the two fluorophores in the absence of glucose was also calculated. FRET pair dyes APTS/TRITC have similar excitation and emission spectra to the commonly paired FITC/TRITC dyes. Therefore, their theoretical Förster radius is expected to be similar, and from the literature, FITC/TRITC is known to have an approximate $R_{0}$ of 5 $\mathrm{nm} .{ }^{26}$ This $R_{0}$ value and the FRET efficiency calculated previously was used to calculate the estimated average distance between the bound and unbound APTS/TRITC based assay via eq 3 and eq 4, which was determined to be 3.5 and $6 \mathrm{~nm}$, respectively, for APTS-MT and APTS-Dex-2M.

\section{Glucose Response of Mannotetraose Based FRET Assay Compared to Dextran}

The performance of these two assays within the physiologically relevant glucose concentration range ( 0 to $400 \mathrm{mg} / \mathrm{dL}$ ) was also evaluated in free solution at room temperature. (Figure 2).

The results showed that the assay containing the fluorescently labeled monovalent ligand, APTS-MT, had a much greater FRET response to changes in glucose concentrations. More 
importantly, APTS-MT was observed to be more accurate in predicting changes in glucose concentrations compared to APTS-Dex-2M. Furthermore, the average sensitivity of the assay to changes in glucose concentration was also calculated by determining the percent change in fluorescence ratio over the total glucose concentration range $(\% \Delta F$.ratio/mM). In free solution, the mannotetraose $(1.3 \pm 0.7 \%)$ based assay is approximately four times more sensitive to changes in physiologically relevant glucose concentrations than APTS-Dex-2 M $(0.35 \pm 0.02 \%)$. Therefore, under the same conditions (i.e., same FRET pair fluorophores and temperature) the competing ligand mannotetraose appeared to have a better predictability and sensitivity to changes in glucose concentration than the dextran (2 M). This may be due to the location of the fluorophore on these molecules. The size of mannotetraose allows the distance between its fluorophore and the fluorophore on the ConA to be in closer proximity than if the same fluorophore was on dextran, which contains multiple long branches terminated with the fluorophore. These multiple long branches therefore result in a less controlled placement of the fluorophore and in turn a less controlled distance between the donor and acceptor fluorophores compared to the mannotetraose based ligand. This in turn affects both the FRET efficiency and sensitivity of the assay resulting in the dextran based assay to be less efficient in predicting glucose concentrations.

The glucose response of APTS-MT based assay was also compared to that of an assay comprising dextran with a MW of $70 \mathrm{kDa}$ (Figure 3). This dextran is the most common dextran reported in the literature for use in ConA-based glucose sensing assays. ${ }^{27}$ In the literature, its binding affinity to ConA is reported to be $\sim 15000 \mathrm{M}^{-1}$. Several research groups have shown this dextran to function within a ConA based glucose sensor with good response to changes in physiological glucose concentrations, both in vitro and in vivo, when a substantial amount of ConA or dextran is used. ${ }^{28}$ However, many have observed sensor failure over time with one possibility of the failure linked to aggregate formation between ConA and dextran. Therefore, previous work has shown that mannotetraose can act as a replacement for this competing ligand with improve functionality due to its nonaggregative behavior when interacting with ConA. ${ }^{20}$

In this work, the glucose response $(0 \mathrm{mg} / \mathrm{dL}$ to $400 \mathrm{mg} / \mathrm{dL})$ of the proposed APTS-MT was also compared to an APTS-Dex-70k assay. However, unlike APTS-Dex-2 M the concentration of mPEG-TRITC-ConA had to be increase substantially in order to observe even the slightest change in FRET in response to glucose. This is due the Dex-70k's low $K_{\mathrm{a}}$ in a ConA based assay. For a dextran 70 based assay, for at least $50 \%$ of its molecules to be in the bound state, $\sim 66 \mu \mathrm{M}$ of ConA (note ConA solubility limit is $100 \mu \mathrm{M}$ ) is required compared to only $1 \mu \mathrm{M}$ for a ConA-mannotetraose assay.

Herein, a mPEG-TRITC-ConA concentration which resulted in at least $25 \%$ of the molecules to initially be bound was used with hopes of minimizing aggregate formation between the two molecules, which is also an issue with these ConA-Dextran based assays. It was observed that its change in fluorescence ratio is greater than that of dextran $2 \mathrm{M}$ with an approximate sensitivity of $0.7 \pm 0.1 \%$ across the same physiological range. Chinnayelka et al. have also reported similar sensitivity of $\sim 0.7 \%$ for a ConA-Dextran 70 based FRET assay in free solution. ${ }^{29-31}$ However, when compared to APTS-MT, the mannotetraose based assay still had improved sensitivity and reproducibility (note the large error bars for APTS- 
Dex-70k assay) in response to changes in glucose concentration overall. The concentration of the competing ligand could have also been increased in these experiments. However, the ideal sensor should be sensitive to lower glucose concentrations, which requires very low concentration of the competing ligand.

\section{Thirty Days Glucose Response of Assay at Body Temperature}

The glucose response of an assay containing mannotetraose at $37^{\circ} \mathrm{C}$ was evaluated after 30 days to determine the assays stability and activity under physiologic thermal conditions (Figure 4). Results show that the MARD\% over the hypoglycemic range ( $<70 \mathrm{mg} / \mathrm{dL}$ ) was $\sim 9 \%$, the euglycemic range (i.e., between 70 and $180 \mathrm{mg} / \mathrm{dL}$ ) was $2 \%$, and the glycocemic range $(<180 \mathrm{mg} / \mathrm{dL}$ ) was $\sim 4 \%$. The overall MARD between 0 and $400 \mathrm{mg} / \mathrm{dL}$ was $\sim 5 \%$.

\section{CONCLUSION}

In this article it was shown that the basic design of the competing ligand, mannotetraose, decreases the average distance between the donor and acceptor when bound. It was also shown that this ligand has improved FRET Efficiency over Dextran-70k and Dextran-2M, the most common competing ligand used in a ConA based glucose assay. Furthermore, over the physiological glucose concentrations ( 0 to $400 \mathrm{mg} / \mathrm{dL}$ ) mannotetraose was shown to be more sensitive to changes in concentration compared to dextran. Finally, it was shown that the assay remains relatively stable after 30 days of being incubated at body temperature $\left(37^{\circ} \mathrm{C}\right)$. Therefore, the monovalent ligand, mannotetraose has the potential to act as an alternate ligand for ConA based glucose sensing assays.

\section{Supplementary Material}

Refer to Web version on PubMed Central for supplementary material.

\section{Acknowledgments}

This work was supported by the National Institutes of Health (R01 DK095101). The authors would like to thank Dr. Michael McShane at Texas A\&M University for use of his laboratory instruments.

\section{References}

1. Klonoff DC. Continuous glucose monitoring roadmap for 21 st century diabetes therapy. Diabetes Care. 2005; 28:1231-1239. [PubMed: 15855600]

2. Tamborlane WV, Beck RW, Bode BW, Buckingham B, Chase HP, Clemons R, Fiallo-Scharer R, Fox LA, Gilliam LK, Hirsch IB. Continuous glucose monitoring and intensive treatment of type 1 diabetes. N Engl J Med. 2008; 359:1464-1476. [PubMed: 18779236]

3. Bode B, Battelino T. Continuous glucose monitoring. Int J Clin Pract. 2010; 64:11-15.

4. Juvenile Diabetes Research Foundation Continuous Glucose Monitoring Study Group. The effect of continuous glucose monitoring in well-controlled type 1 diabetes. Diabetes Care. 2009; 32:13781383. [PubMed: 19429875]

5. Meadows D, Schultz JS. Fiber-optic biosensors based on fluorescence energy transfer. Talanta. 1988; 35(2):145-150. [PubMed: 18964483]

6. Schultz, JS. Optically-based Affinity Biosensors for Glucose. In: Geddes, CD., Lakowicz, JR., editors. Topics in Fluorescence Spectroscopy, Vol 11: Glucose Sensing. Springer; 2006. p. 283-310. 
7. Schultz JS, Mansouri S, Goldstein IJ. Affinity Sensor: A New Technique for Developing Implantable Sensors for Glucose and Other Metabolites. Diabetes Care. 1982; 5:245-253. [PubMed: 6184210]

8. Chinnayelka S, McShane MJ. Glucose sensors based containing an orange/red competitive binding resonance energy transfer assay. Diabetes Technol Ther. 2006; 8:269-278. [PubMed: 16800748]

9. Ballerstadt R, Schultz JS. Competitive-binding assay method based on fluorescence quenching of ligands held in close proximity by a multivalent receptor. Anal Chim Acta. 1997; 345:203-212.

10. Dutt-Ballerstadt R, Evans C, Pillai AP, Orzeck E, Drabek R, Gowda A, McNichols R. A Human Pilot Study of the Fluorescence Affinity Sensor for Continuous Glucose Monitoring in Diabetes. J Diabetes Sci Technol. 2012; 6:362-370. [PubMed: 22538148]

11. Müller AJ, Knuth M, Nikolaus KS, Krivánek R, Küster F, Hasslacher C. First Clinical Evaluatioi of a New Percutaneous Optical Fiber Glucose Sensor for Continuous Glucose Monitoring in Diabetes. J Diabetes Sci Technol. 2013; 7:13-23. [PubMed: 23439156]

12. Nielsen JK, Christiansen JS, Kristensen JS, Toft HO, Hansen LL, Aasmul S, Gregorius K. Clinical Evaluation of a Transcutaneous Interrogated Fluorescence Lifetime- Based Microsensor for Continuous Glucose Reading. J Diabetes Sci Technol. 2009; 3:98-109. [PubMed: 20046654]

13. Pickup JC, Hussain F, Evans ND, Rolinski OJ, Birch DJS. Fluorescence-based glucose sensors. Biosens Bioelectron. 2005; 20:2555-2565. [PubMed: 15854825]

14. Ballerstadt R, Schultz JS. A fluorescence affinity hollow fiber sensor for continuous transdermal glucose monitoring. Anal Chem. 2000; 72:4185-4192. [PubMed: 10994982]

15. Lakowicz, JR. Principles of fluorescence spectroscopy. Springer Science \& Business Media; 2013.

16. Clegg, R. Reviews in Fluorescence 2006. Geddes, C., Lakowicz, J., editors. Springer; New York: 2006. p. 1-45.

17. Ballerstadt R, Evans C, McNichols R, Gowda A. Concanavalin A for in vivo glucose sensing: A biotoxicity review. Biosens Bioelectron. 2006; 22:275-284. [PubMed: 16488598]

18. Ibey BL, Beier HT, Rounds RM, Coté GL, Yadavalli VK, Pishko MV. Competitive Binding Assay for Glucose Based on Glycodendrimer-Fluorophore Conjugates. Anal Chem. 2005; 77:7039-7046. [PubMed: 16255607]

19. Cummins BM, Lim J, Simanek EE, Pishko MV, Coté GL. Encapsulation of a Concanavalin A/ dendrimer glucose sensing assay within microporated poly (ethylene glycol) microspheres. Biomed Opt Express. 2011; 2:1243-1257. [PubMed: 21559135]

20. Cummins BM, Li M, Locke AK, Birch DJ, Vigh G, Coté GL. Overcoming the aggregation problem: A new-type of fluorescent ligand for ConA-based glucose sensing. Biosens Bioelectron. 2015; 63:53-60. [PubMed: 25058939]

21. Locke AK, Cummins BM, Abraham AA, Coté GL. PEGylation of Concanavalin A to improve its stability for an in vivo glucose sensing assay. Anal Chem. 2014; 86:9091-9097. [PubMed: 25133655]

22. Locke, AK., Cummins, BM., Abraham, AA., Coté, GL. SPIE BiOS. International Society for Optics and Photonics; 2015. Long term response of a Concanavalin-A based fluorescence glucose sensing assay.

23. Albalasmeh AA, Asmeret AB, Teamrat AG. A new method for rapid determination of carbohydrate and total carbon concentrations using UV spectrophotometry. Carbohydr Polym. 2013; 97:253-261. [PubMed: 23911443]

24. Findlay JW, Dillard RF. Appropriate calibration curve fitting in ligand binding assay. AAPS J. 2007; 9:E260-E267. [PubMed: 17907767]

25. Benzeval, ID. Development of responsive polymers for drug delivery applications. University Library; 2009.

26. Wu P, Brand L. Resonance energy transfer: methods and applications. Anal Biochem. 1994; 218:113. [PubMed: 8053542]

27. Meadows DL, Schultz JS. Design, manufacture and characterization of an optical fiber glucose affinity sensor based on an homogeneous fluorescence energy transfer assay system. Anal Chim Acta. 1993; 280:21-30.

28. Aloraefy M, Pfefer J, Ramella-Roman J, Sapsford K. Development and testing of a fluorescence biosensor for glucose sensing. Proc SPIE. 2012:83670H. 
29. Chinnayelka S, Zhu H, McShane M. Near-Infrared Resonance Energy Transfer Glucose Biosensors in Hybrid Microcapsule Carriers. J Sens. 2008; 2008:11.

30. Aloraefy M, Pfefer TJ, Ramella-Roman JC, Sapsford KE. In Vitro Evaluation of Fluorescence Glucose Biosensor Response. Sensors. 2014; 14:12127-12148. [PubMed: 25006996]

31. Ballerstadt R, Polak A, Beuhler A, Frye J. In vitro long-term performance study of a near-infrared fluorescence affinity sensor for glucose monitoring. Biosens Bioelectron. 2004; 19:905-914.

[PubMed: 15128110] 

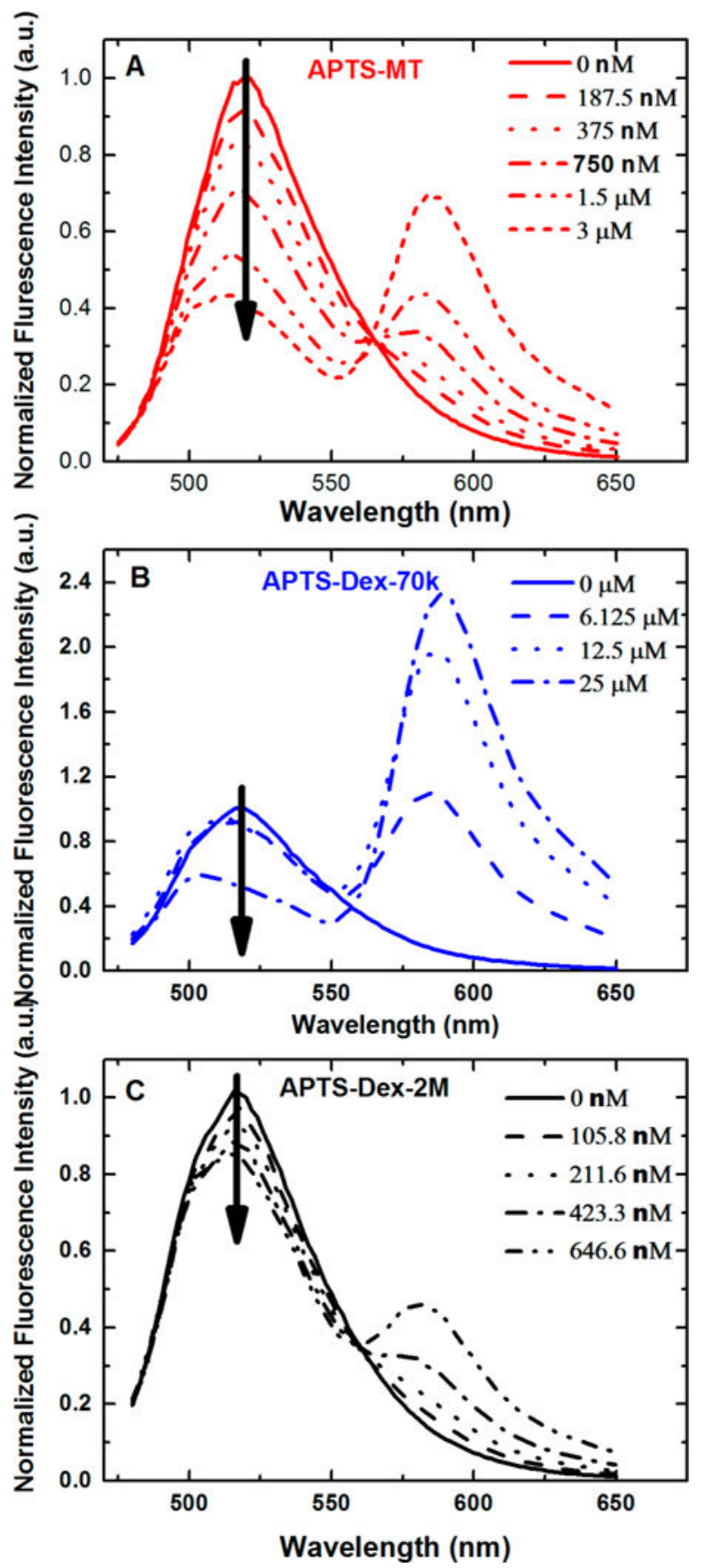

Figure 1.

Titration curves are shown comparing the change in fluorescence intensity of the different donor conjugated ligands: (A) APTS-MT, (B) APTS-Dex-70k, and (C) APTS-Dex-2M, in response to increasing concentration of mPEG-TRITC-ConA (legend). All curves were normalized to the donor peak at $520 \mathrm{~nm}$. 

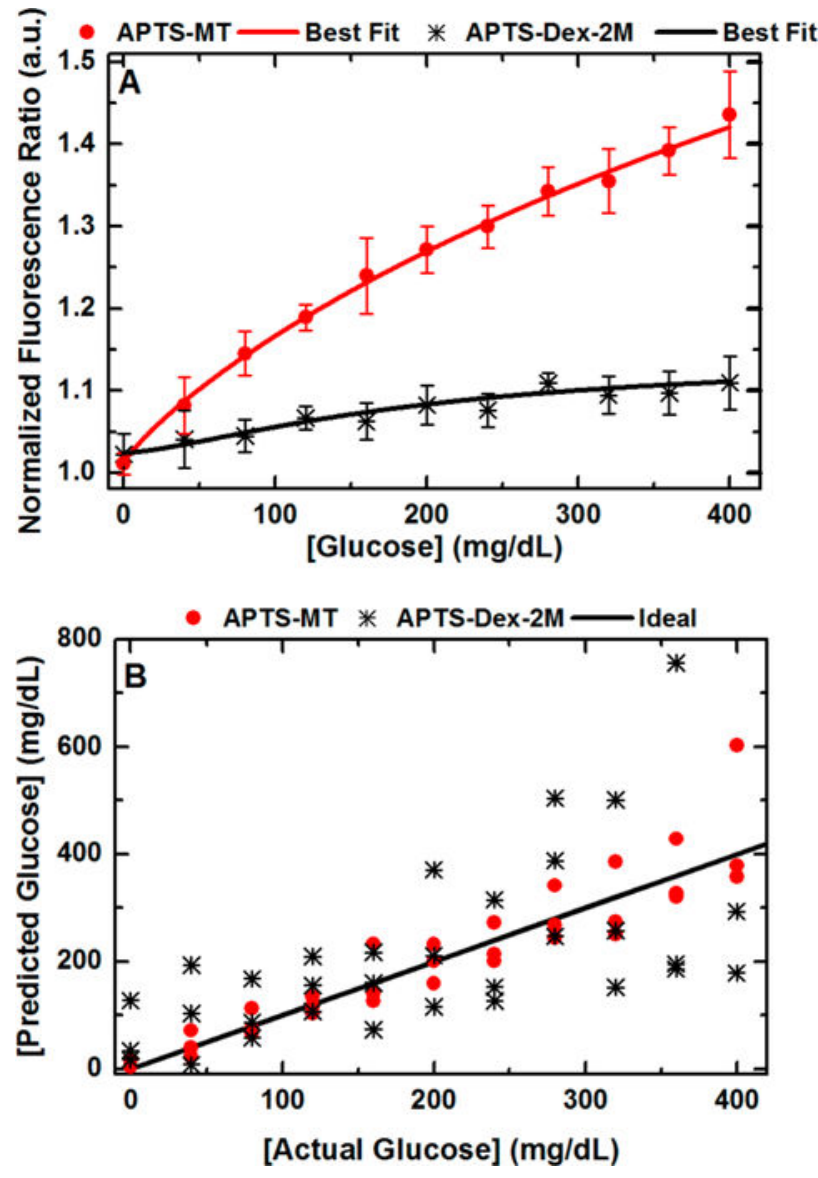

Figure 2.

(A) Average glucose response of assays comprising $200 \mathrm{nM}$ APTS-MT and $1 \mu \mathrm{M}$ mPEGTRITC-ConA showing improved FRET response and (B) predictability of glucose concentrations versus $52 \mathrm{nM}$ APTS-Dex-2 M and $1 \mu \mathrm{M}$ mPEG-TRITC-ConA. Error bars indicate triplicate samples. 

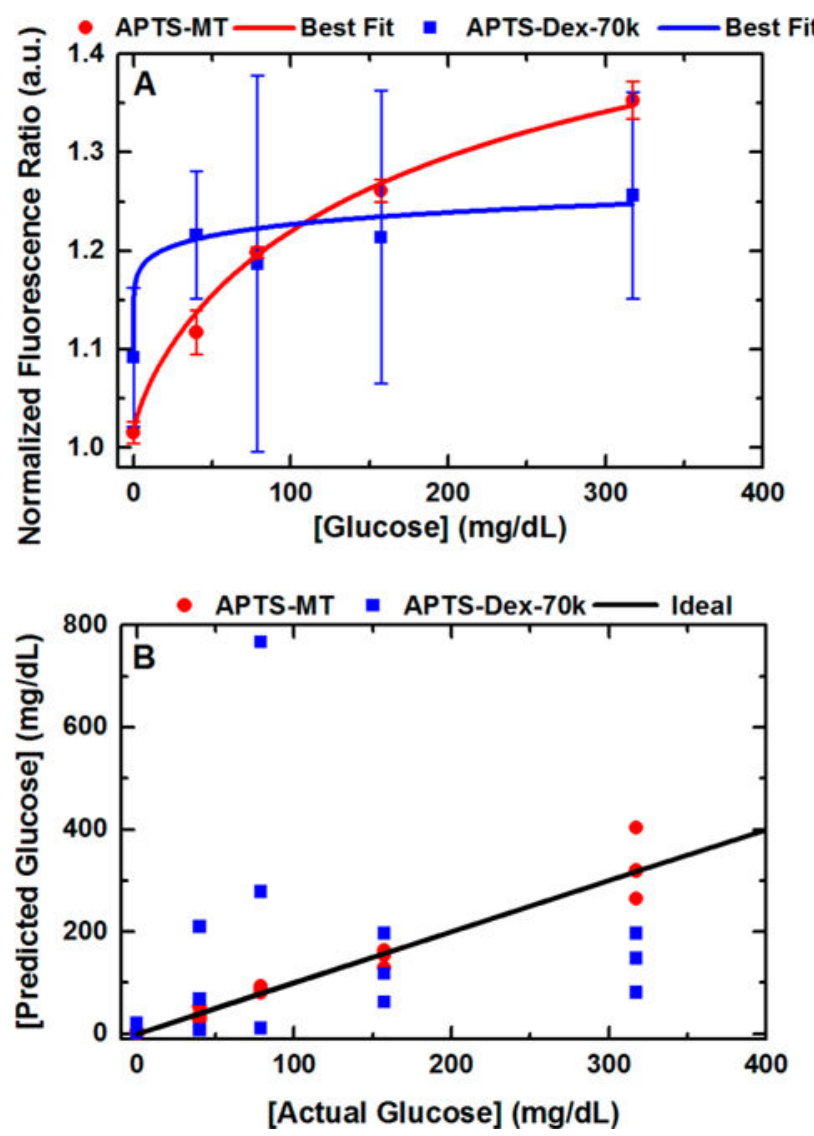

Figure 3.

(A) Average glucose response of assays comprising $200 \mathrm{nM}$ APTS-MT and $1 \mu \mathrm{M}$ mPEGTRITC-ConA showing improved FRET response, reproducibility and (B) predictability of glucose concentrations versus $200 \mathrm{nM}$ APTS-Dex-70k and $25 \mu \mathrm{M}$ mPEG-TRITC-ConA. Error bars indicates triplicate samples. 

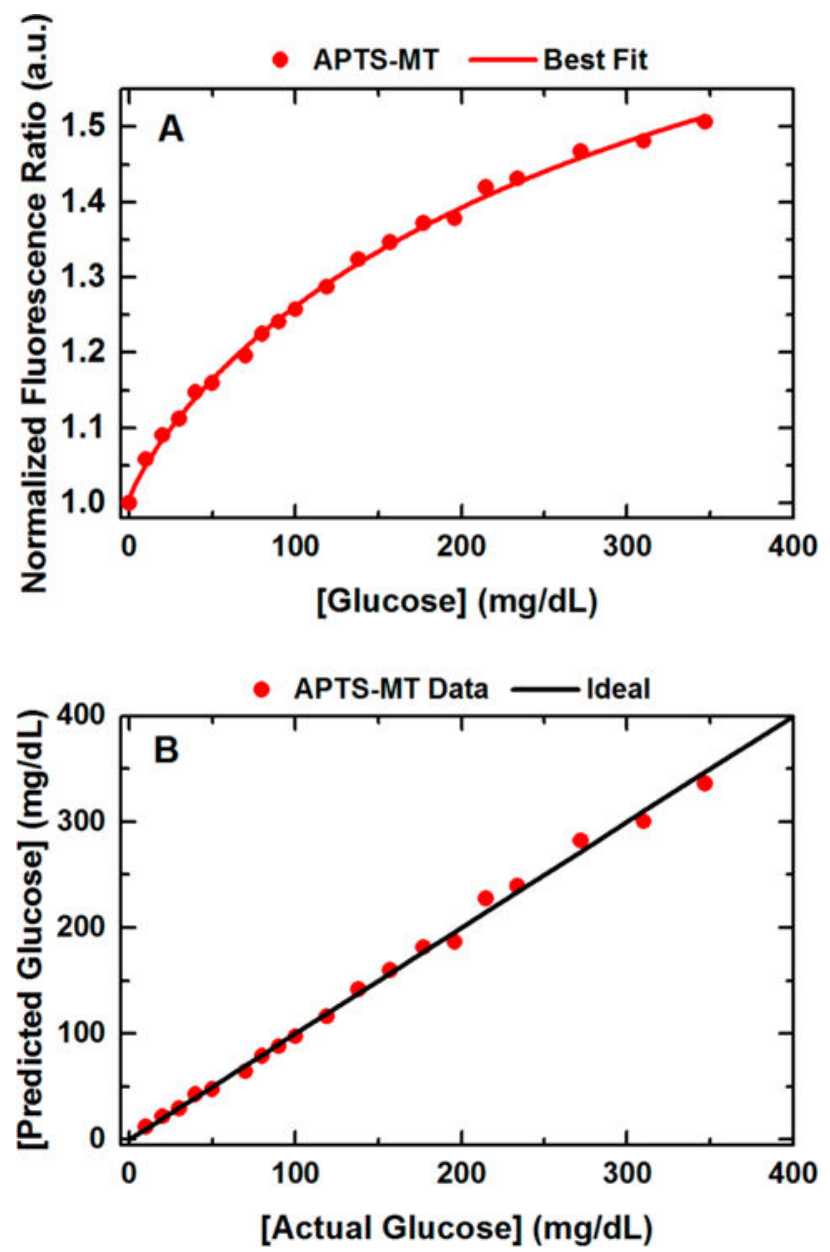

Figure 4.

(A) Normalized fluorescence ratio of assay composed of ConA-MT and (B) predicted versus actual glucose response of the same assay indicating the assay's ability to predict changes in glucose concentrations with a MARD of less than $10 \%$ after 30 days incubation at $37{ }^{\circ} \mathrm{C}$. 